\title{
Factores Socioeconómicos de la Calidad de Vida de los Adultos Mayores en la Provincia de Guayas, Ecuador
}

\author{
Miguel A. Bustamante ${ }^{(1,2) \star}$, María del C. Lapo ${ }^{(2)}$, José D. Torres ${ }^{(3)}$, Segundo M. Camino ${ }^{(4)}$ \\ (1) Facultad de Economía y Negocios, Universidad de Talca, Campus Lircay. Avenida Lircay S/N, Talca, \\ Chile. (e-mail: mabu@utalca.cl) \\ (2) Facultad de Ciencias Económicas y Administrativas y del Sistema de Posgrado, Universidad Católica de \\ Santiago de Guayaquil, Av. Carlos Julio Arosemena Km. 11⁄2 vía Daule, Guayaquil, Ecuador. \\ (e-mail: maria.lapo@cu.ucsg.edu.ec) \\ (3) Universidad Católica de Santiago de Guayaquil, Programa de Maestría en Gerencia de Servicios de la \\ Salud, Guayaquil, Ecuador (e-mail: jose.torres06@cu.ucsg.edu.ec) \\ (4) Facultad de Ciencias Económicas y Administrativas, Universidad Católica de Santiago de Guayaquil, Av. \\ Carlos Julio Arosemena Km. 11⁄2 vía Daule, Guayaquil, Ecuador. (e-mail: segundo.camino@cu.ucsg.edu.ec)
}

${ }^{*}$ Autor a quien debe ser dirigida la correspondencia.

Recibido Ene. 30, 2017; Aceptado Abr. 13, 2017; Versión final May. 9, 2017, Publicado Oct. 2017

\section{Resumen}

El presente trabajo analiza los principales determinantes socioeconómicos de la calidad de vida de los adultos mayores en la provincia del Guayas en Ecuador. Se aplicó el método de encuesta a una muestra de 817 entrevistados el año 2015. Se realizó un análisis descriptivo demográfico y socioeconómico y, para la operación de variables, se generaron variables categóricas y dicotómicas dummy. Se realizó un análisis de factores de componentes principales con rotación Varimax y se modeló alrededor de una variable latente en la forma de un logit binomial. Se concluye que el análisis de factores no resultó satisfactorio en términos de confiabilidad aunque mostró índices KMO adecuados. El análisis logit ordenado permite inferir que el área de residencia no es determinante de la calidad de vida de los adultos mayores. Sin embargo, esta juega un papel importante cuando se analizan diferencias entre hombres y mujeres. La ocupación y el nivel de educación muestran una asociación estadísticamente significativa con la calidad de vida auto-reportada y que el hecho de padecer una enfermedad y pertenecer al rango de edad de entre 71 y 80 años, tendría un impacto negativo en la calidad de vida de los adultos mayores.

\section{Socio-Economic Factors of the Quality Of Life of Older Adults in the Province of Guayas, Ecuador}

\begin{abstract}
The present work analyses the main socioeconomic factors that determine the quality of life of older adults in the Province of Guayas in Ecuador. The survey method was used on a sample of 817 persons interviewed during 2015. A descriptive demographic and socioeconomic analysis was carried out and dummy categorical and dichotomous variables were generated for the operation on variables. A principal components factor with Varimax rotation analysis was performed and it was modelled around a latent variable in the form of a binomial Logit. It was found that the analysis of factors was not satisfactory in terms of reliability, although it showed adequate KMO indices. The ordered logit analysis shows that the residence area is not determinative of the quality of life of older adults, but it does play an important role when analysing differences between men and women. The occupation and level of education show a statistically significant association with the self-reported quality of life, and the fact of suffering from diseases and belonging to the 71-80 age range may have a negative impact on the quality of life of older adults.
\end{abstract}

Keywords: quality of life; older adult; health economics; aging; sociodemographic determinants 


\section{INTRODUCCIÓN}

Los países de América Latina y del Caribe (ALC) duplicarán la cantidad de adultos mayores (AM) y representará 16,7\% de la población (CEPAL, 2014). En Ecuador, la población mayor de 60 años de edad llegó en 2015 a un 10\% del total (United Nations, 2015) y se incrementaría a un 13\% en 2025 y al $26 \%$ en el 2060 (CEPAL, 2015). Según el censo de 2010 este grupo poblacional en el Guayas era de 940.905 lo que representaba el 6,6\%, al año 2014 y que para el 2017 se estima en 1.180 .944 equivalente a un $7,0 \%$ de la población total, siendo la provincia del Guayas la con mayor número de adultos mayores a la fecha (CNII, 2014).

En lo conceptual, la calidad de vida (QoL) ha sido utilizada como una medida de bienestar y aunque no existe una definición concordada, en la mayoría de los casos se mal emplea (Urzúa y Caqueo-Urízar, 2012). El concepto surge de la percepción multidimensional que tienen los individuos acerca de su vida, sus expectativas y proyectos de largo plazo e incluye estilo de vida, vivienda, satisfacción en la escuela y en el trabajo, así como su situación económica, entre otros (Quintana y Paravic, 2014). La tendencia a investigar la calidad de vida implica no sólo estudiar cómo satisfacer necesidades sino cómo mejorarla actuando sobre dimensiones objetivas y subjetivas de la misma (Guedes y Martínez 2010). En término de los factores objetivos, la encuesta mundial realizada por Gallup en el año 2006 muestra una correlación positiva entre el ingreso per cápita y la satisfacción con la calidad de vida, sin embargo, "no hay evidencia que los efectos entre países de mayores ingresos desaparezcan o desvanezcan por un incremento de la renta de los países" (Deaton, 2008).

Aunque, la calidad de vida está condicionada por factores sociales, individuales, de salud física y material (Osorio et al. 2011), no se concluye en una definición de calidad de vida relacionada a la salud (Urzúa, 2010) que resulte unívoca, al parecer, porque el estado de salud y calidad de vida son conceptos que se confunden o bien, porque calidad de vida no se aísla de normas culturales, patrones de conducta y expectativas lográndose sólo aproximaciones parciales a un concepto calidad de vida en referencia a un nivel de bienestar que proviene de diversos dominios de la vida y que se expresa en el estado de salud (Quintana y Paravic, 2014; Guedes y Martínez 2010).

En el caso de Ecuador en 2010, la calidad de vida de los adultos mayores indicaba que cerca del $23 \%$ vivía en condiciones sociales buenas, el $54 \%$ en condiciones regulares o malas y un $23 \%$ en condiciones de indigencia (Freire et al., 2010). Ademas, los factores socio-demográficos tales como edad, género, estado civil, entre otros y, la situación laboral, el padecer o no alguna enfermedad, tienen efecto en la autosatisfacción de la calidad de vida, confirmando que el grado de satisfacción con la vida está determinado por las características individuales de cada persona como edad, género, estado marital, grado de satisfacción con su salud y, por las características específicas ambientales que se viven en cada país (Hritcu, 2015), de modo que la satisfacción con la vida es un indicador de bienestar que incluye la salud percibida y que conlleva un sentimiento de salud física y mental, asociado con una mayor longevidad (Mincu y Tascu, 2015).

En consecuencia, la calidad de vida relacionada a la salud evalúa diversas dimensiones (Urzúa et al, 2011) como son los resultados de intervenciones médicas e impacto de enfermedades cotidianas mediante estudios de percepción de los afectados, respecto de bienestar físico, sicológico, social y espiritual de personas insertas en un entorno cultural y de historia colectiva (Urzúa, 2010). En términos de los factores subjetivos de calidad de vida, el trato constante con colegas y amigos reduce las discapacidades al igual que el tiempo libre y las relaciones de amistad cercanas más no la presencia de los familiares (Hritcu, 2015) y se ratifica que el trato frecuente con al menos cinco amigos, ayuda a alcanzar un envejecimiento exitoso, es decir, permite a las personas llegar a convertirse en AM sin discapacidad alguna (Urzúa, 2010), confirmando que las relaciones de apoyo interpersonal tienen un gran valor al mantener la salud, bienestar y reducir el estrés.

Es así como, asumiendo que la salud es un derecho elemental del ser humano y un factor insustituible que influye en el bienestar social, el desarrollo económico y la educación de las naciones se ubican a la base de estas necesidades (Knoepfel et al., 2012; Malagón et al., 2008). En lo relativo a la salud, los AM concentran la mayor cantidad de consumidores de servicios médicos y medicinas, situación que desde el punto de vista del Estado, representa una fuerte inversión en lo que respecta a seguridad social y pensiones jubilares (Alonso et al. 2007).

En el Ecuador, existen leyes y lineamientos que exigen una atención especial para los grupos vulnerables como son los adultos mayores, los niños y adolescentes, mujeres embarazadas y personas con discapacidad, personas privadas de la libertad y aquellos que padezcan enfermedades catastróficas, los cuales son resguardados por la Constitución de Ecuador (2008). Por una parte, porque la salud es un 
atributo multidimensional conformando un concepto latente que captura aspectos subjetivos (salud autopercibida, bienestar, calidad de vida, felicidad, satisfacción con la vida), clínicos (morbilidad, declinación funcional) y biológicos (biomarcadores) (Hyland et al., 2014) y, por otra, porque la salud es auto-percibida por las personas lo mque les permite definir una calidad de vida con respeto a la salud (HRQoL) incluyendo aspectos físicos, mentales y sociales (Solís et al., 2016).

Conforme lo indicado y sobre la base de los fundamentos conceptuales que definen la satisfacción con la vida y, cómo ésta se relaciona con los componentes subjetivos de la calidad de vida, el presente trabajo analiza los principales determinantes socioeconómicos de la calidad de vida de los adultos mayores en la provincia del Guayas - Ecuador.

\section{METODOLOGÍA}

El diseño de la investigación es de corte transversal al año 2015, la población objetivo está constituida por adultos mayores, AM de ambos sexos mayores o iguales a 60 años institucionalizados en Centros Gerontológicos, que residen en áreas urbanas y rurales de la provincia del Guayas, Ecuador (Hernández et al, 2006). Se realizó un muestreo por conglomerados y estratificado conformando una muestra de 817 personas y se realizó una evaluación de las variables demográficas, sexo, edad, estado civil, etnia, educación, ocupación, fuente de ingreso, zona de residencia y auto satisfacción con la vida. Las respuestas a los reactivos se solicitaron vía escalas cualitativas.

Como estratos se utilizaron los 25 cantones de la provincia de Guayas, con afijación proporcional al tamaño de la población muestreada. Dentro de cada cantón se realizó la afijación por conglomerado, también de forma proporcional al tamaño de la población muestreada (Eyssautier, 2006; Hernández, et al. 2006). El estrato quedó finalmente como la intersección de comuna y conglomerado. Como conglomerados se utilizaron los centros gerontológicos seleccionados mediante un muestreo aleatorio simple, con la función "muestra aleatoria de casos" de SPSS. Dado el número de casos recogidos, la muestra del presente estudio dio origen a un modelo correctamente especificado para cumplir con las propiedades de insesgadez y consistencia recomendadas en la literatura que aborda el modelamiento logit (Guarín, et al. 2012).

\section{Definición de Variables}

Se trabajó con una variable dependiente discreta (Rodríguez et al 2007; Greene, 2003) que mide cualidades mediante un número finito de alternativas de auto-satisfacción de los individuos (Osorio et al. 2011) a través de la pregunta "Respecto a su propia vida, usted está en general...", teniendo los encuestados que escoger entre distintas opciones de respuesta categorizadas en 4 niveles: 1) si el individuo está altamente insatisfecho, 2) si se encuentra un poco insatisfecho, 3) si está un poco satisfecho y 4) si está altamente satisfecho. Para la operación de variables se recurrió a variables categóricas y a la generación de variables dicotómicas Dummy (Walpole et al., 2007) susceptibles de ser modelizadas utilizando técnicas econométricas (Greene, 2003). La Tabla 1 presenta un detalle de las variables empleadas.

Conceptualmente, entre las Dummy se mencionan variables indicativas, categóricas, dicótomas, binarias, ficticias o estacionales, cualitativas, categóricas o de control, en consecuencia, se trabajó con variables categóricas y Dummy (Rodríguez et al 2007; Greene, 2003) relacionando la variable dependiente con las independientes cuantitativas o cualitativas, asumiendo que las variables Dummy determinan la presencia 0 ausencia de una cualidad o un atributo (Greene, 2003) que, en su carácter de variables explicativas, se incorporan al análisis como variables de control, indicadoras o pseudo-variables.

En consecuencia y, dado que las variable Dummy pueden derivar de variables con categorías, estas se modelan como cualitativas a fin de evitar la trampa de la variable dicótoma como es la multicolinealidad perfecta (Guarín, et al. 2012; Rodríguez et al 2007; Greene, 2003).

Tabla 1: Variables utilizadas en el modelo

\begin{tabular}{|l|l|l|l|}
\hline \multicolumn{1}{|c|}{ Nombre/Tipo } & \multicolumn{1}{|c|}{ Descripción } & Nombre/Tipo & \multicolumn{1}{c|}{ Descripción } \\
\hline $\begin{array}{l}\text { Auto-satisfacción de } \\
\text { los individuos con su } \\
\text { propia vida } \\
\text { Categórica }\end{array}$ & $\begin{array}{l}\text { Altamente Satisfecho } \\
\text { Un poco satisfecho } \\
\text { Un poco insatisfecho } \\
\text { Altamente insatisfecho }\end{array}$ & $\begin{array}{l}\text { Edad } \\
\text { Categórica }\end{array}$ & $\begin{array}{l}\text { 60-70 años (categoría omitida) } \\
71-80 \text { años (grupo_edad2) } \\
81+\text { años (grupo_edad3) }\end{array}$ \\
\hline $\begin{array}{l}\text { Estado civil } \\
\text { Categórica }\end{array}$ & $\begin{array}{l}\text { Soltero (estado_civil1) } \\
\text { Divorciado o separado } \\
\text { (estado_civil2) }\end{array}$ & $\begin{array}{l}\text { Grupo étnico } \\
\text { Categórica }\end{array}$ & $\begin{array}{l}\text { Mestizo (categoría omitida) } \\
\text { Afroamericano (auto_etnia2) } \\
\text { Montubio (auto }\end{array}$ \\
\hline
\end{tabular}


Tabla 1 (continuación)

\begin{tabular}{|l|l|l|l|}
\hline $\begin{array}{l}\text { Nivel educativo } \\
\text { Categórica }\end{array}$ & $\begin{array}{l}\text { Ninguno (nivel_educ1) } \\
\text { Básica (nivel_educ2) } \\
\text { Bachillerato (nivel_educ3) } \\
\text { Tercer nivel } \\
\text { (categoría omitida) }\end{array}$ & $\begin{array}{l}\text { Ocupación } \\
\text { Categórica }\end{array}$ & $\begin{array}{l}\text { Cuenta propia (categoría omitida) } \\
\text { Empresario (Labor72) } \\
\text { Particular (Labor73) } \\
\text { Público (Labor74) } \\
\text { Jubilado (Labor75) } \\
\text { Buscando trabajo (Labor76) } \\
\text { En otra situación (Labor77) }\end{array}$ \\
\hline $\begin{array}{l}\text { Sexo } \\
\text { Dummy }\end{array}$ & $\begin{array}{l}\text { Toma el valor de 1 si el individuo } \\
\text { es mujer y 0 si es hombre }\end{array}$ & $\begin{array}{l}\text { Principal fuente } \\
\text { de ingreso } \\
\text { Dummy }\end{array}$ & $\begin{array}{l}\text { Toma el valor de 1 si el individuo es } \\
\text { el principal sustento económico } \\
\text { (principal) y 0 caso contrario. }\end{array}$ \\
\hline $\begin{array}{l}\text { Enfermedad } \\
\text { específica } \\
\text { Dummy }\end{array}$ & $\begin{array}{l}\text { Toma el valor de 1 si el individuo } \\
\text { padece de una o más } \\
\text { enfermedades del listado } \\
\text { (enfermedad) y 0 caso contrario. }\end{array}$ & $\begin{array}{l}\text { Área de } \\
\text { residencia } \\
\text { Dummy }\end{array}$ & $\begin{array}{l}\text { Toma el valor de 1 si el individuo } \\
\text { reside en la zona urbana (urbano) y 0 } \\
\text { caso contrario. }\end{array}$ \\
\hline
\end{tabular}

\section{Análisis de factores de componentes principales}

Con el objetivo de determinar la eventual concentración de componentes en factores relevantes, se realizaron tres pruebas con rotación varimax.

La primera consideró la totalidad de las variables en estudio, determinando un índice $\mathrm{KMO}$ de 0,586, una confiabilidad mínima para los componentes tipificados de 0,132 y una concentración en 7 factores que explican un $55,256 \%$ de la varianza total. El segundo análisis incluyó el listado de 10 enfermedades y la variable satisfacción con la vida dando como resultados un índice KMO de 0,605 , una confiabilidad para los componentes tipificados de 0,203 y una concentración en 4 factores que explican un $48.403 \%$ de la varianza total. Finalmente, la tercera prueba analizó las variables demográficas y la variable satisfacción con la vida dando como resultados un índice KMO de 0,617, una confiabilidad de 0,272 y una concentración en 4 factores que explican un $67,686 \%$ de la varianza total. En consecuencia, estos análisis fueron descartados para el estudio de los datos con esta metodología.

\section{Modelo de estimación}

El método de estimación asume que la calidad de vida de los adultos mayores no puede ser medida de manera directa, en consecuencia, se la denomina variable latente y su valor depende de otras variables observadas o manifiestas, las que sí pueden ser medidas a través de preguntas. En este trabajo, se midió por medio de la variable latente discreta dependiente de carácter ordinal "Respecto a su propia vida, usted está en general..." con cuatro categorías de respuesta donde la categoría más alta es "Altamente satisfecho" mientras que la categoría más baja es "Altamente insatisfecho" que se analiza en relación con un conjunto de otras variables observadas o manifiestas con las cuales se modela el análisis.

Se tuvo en consideración los modelos Logit o Probit ordenados y ante la evidencia que los resultados que se obtienen en ambos casos resultan similares (Penna, 2010; Penna, 2006), en el presente trabajo se optó por el modelo de regresión Logit ordenado, que permite modelar variables cualitativas a partir de una función de distribución logística aplicable a una variable dependiente discreta constituida por más de dos categorías (Walpole et al., 2007; Fosgerau y Bjorner, 2006; Boz y Akbay, 2005; Choo et al. 2005; Ndejunga y Nelson, 2005; Greene, 2003).

Entre las variables independientes explicativas o manifiestas, se consideraron las demográficas sexo, edad, estado civil, etnia, educación, ocupación, fuente de ingreso, zona de residencia, enfermedad específica y, como variable dependiente, la auto satisfacción con la vida. Se tuvo a la vista, además, que para este tipo de variable dependiente, un modelo logit multinomial no sería capaz de considerar la naturaleza ordinal de la variable (Fosgerau y Bjorner, 2006; Boz y Akbay, 2005; Choo et al. 2005, Ndejunga y Nelson, 2005) y que los modelos ordinarios de regresión tampoco serían los adecuados, porque un "ranking" ordinal de diferencias inter-escales no son comparables, sin embargo, el modelo logit ordenado resultó ser el más adecuado para modelar la pregunta de interés (Penna, 2010).

El modelo logit ordenado se construyó alrededor de una variable latente en la misma forma que un Logit binomial (Walpole et al., 2007; Hernández et al., 2006; Greene, 2003) asumieniendo que en este tipo de modelos, conforme a su definición, la variable dependiente representa la probabilidad de que ocurra el fenómeno que se está analizando. 
El modelo genérico queda definido de la siguiente forma:

$$
y^{*}=x^{\prime} \beta+\varepsilon,
$$

Donde al igual que en los modelos logit binomiales $y^{\star}$ no es directamente observable. Lo que se observa es:

$$
\begin{aligned}
& y=0 \text { si } y^{*} \leq 0, \\
& y=1 \text { si } 0<y^{*} \leq \mu_{1}, \\
& y=2 \text { si } \mu_{1}<y^{*} \leq \mu_{2}, \\
& y=J \text { si } \mu_{j-1} \leq y^{*}
\end{aligned}
$$

En la ecuación (5), J constituye el número de posibles respuestas de la variable latente discreta dependiente de carácter ordinal y categórica. En este caso particular $\mathrm{J}$ define las posibles respuestas de los individuos encuestados en la pregunta "Respecto a su propia vida, usted está en general...." tienen 4 posibles respuestas tal como se las categorizo en los párrafos anteriores. Los $\mu_{\mathrm{s}}$ son parámetros desconocidos que serán estimados en conjunto con los $\beta$ (coeficientes) de la regresión y como resultados del estudio para la consecuente interpretación. Finalmente, $\mathrm{J}$ es igual a 4, quedando las expresiones como se indica en la formulación 6 a 9 siguientes.

$$
\begin{aligned}
& y=1 \text { si } y^{*}<\mu_{1}, \\
& y=2 \text { si } \mu_{1}<y^{*}<\mu_{2}, \\
& y=3 \text { si } \mu_{2}<y^{*}<\mu_{3}, \\
& y=4 \text { si } y^{*}>\mu_{3}
\end{aligned}
$$

Para un modelo Logit, que sigue una distribución logística, en el caso de dos variables independientes, discretas y constituidas por más de dos categorías, las probabilidades de ocurrencia de cada una de las categorías viene dada por:

$P\left(y=j \mid x_{1}, x_{2}\right)=\frac{\exp \left(r_{j}-\beta_{1} x_{1}-\beta_{2} x_{2}\right)}{1+\exp \left(r_{j}-\beta_{1} x_{1}-\beta_{2} x_{2}\right)}-\frac{\exp \left(r_{j-1}-\beta_{1} x_{1}-\beta_{2} x_{2}\right)}{1+\exp \left(r_{j-1}-\beta_{1} x_{1}-\beta_{2} x_{2}\right)}$

Para la estimación del modelo econométrico (Fosgerau y Bjorner, 2006; Boz y Akbay, 2005) se introdujeron adicionalmente dos términos de interacción: (i) entre el sexo y residentes en el área urbana para el evaluar la asociación que pudiese existir entre mujer-urbano y la autosatisfacción con la vida; y (ii) entre el padecer o no una enfermedad específica con residir en el área urbana para así mismo, evaluar la asociación que pudiese existir entre enfermedad-urbano y la autosatisfacción con la vida. Para ello se modelizaron las variables como dummy de manera que, cuando la variable latente supera un determinado nivel, la variable discreta toma el valor 1, y si no lo supera toma el valor 0 (Greene, 2003).

Se tuvo en consideración que, si los resultados se distribuyen como una normal con media cero y varianza uno, el modelo generado será un Probit pero, si se distribuyen como una curva o función de distribución logística, se trataría de un modelo Logit Ordenado (Penna, 2010; Penna, 2006), que se estima por máxima verosimilitud y no por mínimos cuadrados ordinarios, entonces la relación que existe entre las variables explicativas y la probabilidad de ocurrencia del fenómeno analizado, no es lineal.

La relación lineal es con la variable índice, inobservable latente $\mathrm{y}^{*}$ (variable dependiente), en consecuencia, los coeficientes estimados no muestran directamente la magnitud sino únicamente la dirección en la que se mueve la probabilidad en referencia con la categoría más alta (Penna, 2010; Greene, 2003). En consecuencia, para obtener una estimación de la magnitud de las asociaciones en referencia a cualquiera de las categorías, se calculan los efectos marginales promedios que muestra la probabilidad de ocurrencia de cualquiera de las categorías de la variable dependiente (Walpole et al., 2007; Hernández et al., 2006; Greene, 2003).

Finalmente, es preciso indicar que la estimación con efectos marginales promedio, mejora el tipo de análisis vía comparación con alguna otra variable y de esta manera contrastarla (Penna, 2010; Penna, 2006). Para este caso se buscó comparar la calidad de vida de los adultos mayores respecto a su lugar de residencia: urbana y rural. De esta forma se determinó cuáles serían los adultos mayores con mejor o peor calidad de vida respecto a un lugar determinado de residencia. Para el tratamiento de los datos y análisis de los resultados se utilizó el software STATA 13. 


\section{RESULTADOS}

La Tabla 2 muestra que la mayor proporción de adultos mayores residen en la ciudad de Guayaquil $(40,14 \%)$ seguido la ciudad de Milagros $(21,41 \%)$. Las demás ciudades muestran una participación decreciente que va de 5,01\% para los residentes de Daule a 0,12\% para quienes residen en Isidro Ayora.

Tabla 2: Cobertura de la encuesta

\begin{tabular}{|l|c|c|l|c|c|}
\hline Comuna & Total & $\%$ & Comuna & Total & $\%$ \\
\hline Guayaquil & 328 & 40,14 & Playas & 6 & 0,73 \\
\hline Milagro & 175 & 21,41 & Santa Lucía & 6 & 0,73 \\
\hline Daule & 41 & 5,01 & El Triunfo & 5 & 0,61 \\
\hline Salitre & 36 & 4,40 & Balzar & 4 & 0,48 \\
\hline Yaguachi & 36 & 4,40 & Juján & 3 & 0,36 \\
\hline Naranjal & 35 & 4,28 & Naranjito & 3 & 0,36 \\
\hline El Empalme & 31 & 3,79 & Balao & 2 & 0,24 \\
\hline Pedro Carbo & 22 & 2,69 & Lomas de Sargentillo & 2 & 0,24 \\
\hline Samborondón & 22 & 2,69 & Nobol & 2 & 0,24 \\
\hline Durán & 21 & 2,57 & Palestina & 2 & 0,24 \\
\hline Otros & 15 & 1,83 & Crnel. Marcelino Maridueña & 1 & 0,12 \\
\hline Colimes & 9 & 1,10 & Isidro Ayora & 1 & 0,12 \\
\hline Simón Bolívar & 9 & 1,10 & & & \\
\hline Total General & & & & 817 & $100 \%$ \\
\hline
\end{tabular}

De la Tabla 3 se observa que existen más hombres (52,51\%) que mujeres dentro de los encuestados. Además, en cuanto a la ocupación reportada, se tiene que una importante proporción de la muestra se declara jubilados $(24,11 \%)$ y empleados dentro del sector privado $(29,13 \%)$. Por otro lado, un elevado $63,28 \%$ declara no ser la principal fuente de ingreso del hogar.

Tabla 3: Estadísticos descriptivos de las variables

\begin{tabular}{|c|c|c|c|c|c|c|c|}
\hline \multirow{16}{*}{$\begin{array}{l}\text { Variables } \\
\text { independientes }\end{array}$} & Sexo & $\%$ & \multicolumn{2}{|l|}{ Edad } & $\%$ & Estado civil & $\%$ \\
\hline & Mujer & 47,49 & \multicolumn{2}{|l|}{ 60-70 años } & 41,25 & Soltero & 9,30 \\
\hline & Hombre & 52,51 & \multicolumn{2}{|l|}{ 71-80años } & 40,27 & $\begin{array}{l}\text { Divorciado } \\
\text { separado }\end{array}$ & 13,71 \\
\hline & & & \multicolumn{2}{|l|}{$81+$ años } & 18,48 & Viudo & 31,46 \\
\hline & & & & & & Casado & 45,53 \\
\hline & Grupo étnico & $\%$ & \multicolumn{2}{|c|}{ Nivel Educativo } & $\%$ & Ocupación & $\%$ \\
\hline & Mestizo & 58,51 & \multicolumn{2}{|c|}{ Ninguno } & 13,95 & Cuenta propia & 25,21 \\
\hline & Afroamericano & 3,18 & \multicolumn{2}{|l|}{ Básica } & 53,12 & Empresario & 1,35 \\
\hline & Montubio & 32,31 & \multicolumn{2}{|l|}{ Bachillerato } & 20,56 & Particular & 2,57 \\
\hline & Indígena & 5,63 & \multicolumn{2}{|l|}{ Tercer nivel } & 12,36 & Público & 0,24 \\
\hline & Blanco & 0,37 & & & Jubilado & 24,11 \\
\hline & \multirow{2}{*}{\multicolumn{5}{|c|}{ 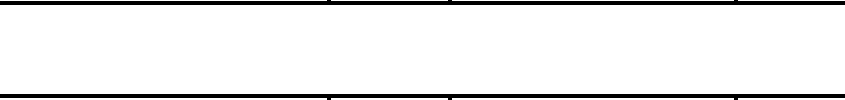 }} & Buscando trabajo & 1,35 \\
\hline & & & & & & En otra situación & 45,17 \\
\hline & $\begin{array}{l}\text { Principal fuente de } \\
\text { ingreso }\end{array}$ & $\%$ & \multicolumn{2}{|l|}{$\begin{array}{l}\text { Enfermedad } \\
\text { específica }\end{array}$} & $\%$ & Área de residencia & $\%$ \\
\hline & Sí & 36,72 & \multicolumn{2}{|l|}{ Sí } & 94,98 & Rural & 49,94 \\
\hline & No & 63,28 & \multicolumn{2}{|l|}{ No } & 5,02 & Urbana & 50,06 \\
\hline \multirow{3}{*}{$\begin{array}{l}\text { Variable } \\
\text { dependiente }\end{array}$} & \multicolumn{4}{|c|}{ Auto-satisfacción con la vida } & & $\%$ \\
\hline & \multicolumn{2}{|l|}{ Altamente insatisfecho } & & 2,69 & \multicolumn{2}{|c|}{ Un poco satisfecho } & 38,68 \\
\hline & \multicolumn{2}{|l|}{ Un poco insatisfecho } & & 4,65 & \multicolumn{2}{|c|}{ Altamente satisfecho } & 53,98 \\
\hline
\end{tabular}

Una primera interpretación, muestra que al parecer, los ingresos no son tan importantes para determinar la calidad de vida de los AM. Respecto del área de residencia la distribución de los encuestados es similar, con un $49,94 \%$ para el área rural y un $50,06 \%$ para la zona urbana. 
Con respecto al padecimiento de enfermedades, la Tabla 4 muestra que de las 10 enfermedades incluidas en el cuestionario, casi la totalidad de los encuestados declara padecer una o más de estas enfermedades con un $94,98 \%$ de representación. Sin embargo, se observa que la mayor parte de la muestra reporta estar altamente satisfecho con su propia calidad de vida $(53,98 \%)$.

Tabla 4: Enfermedades que padecen los adultos mayores del Guayas - Ecuador.

\begin{tabular}{|l|c|l|c|}
\hline Enfermedades & $\%$ & Enfermedades & $\%$ \\
\hline Cáncer & 1,96 & Golpes & 9,06 \\
\hline Respiratoria & 3,92 & Gastrointestinales & 10,76 \\
\hline Hipertensión & 5,39 & Hígado/Vesícula & 12,00 \\
\hline Corazón & 5,63 & Renales & 13,59 \\
\hline Diabetes & 8,20 & Reumatismo & 24,47 \\
\hline Ninguna & 5,02 & & 100,00 \\
\hline Total & & & \\
\hline
\end{tabular}

\section{Modelamiento Econométrico}

Los resultados del modelo econométrico se presentan en la Tabla 5. Para la obtención de resultados, se tuvo en consideración los efectos marginales promedios para la categoría altamente insatisfecho (categoría más baja, donde dy/dx para los niveles de factor es el cambio discreto desde el nivel base, * significativo al $10 \%,{ }^{* * *}$ significativo al $\left.1 \%\right)$.

En general se observa que siete de las variables independientes analizadas muestra resultados significativos $\left({ }^{*},{ }^{* * *}\right)$ en relación con la calidad de vida representada en la variable dependiente satisfacción con la vida, asumiendo que $p$ es una medida de la evidencia que aporta a la interpretación de los datos a favor de la hipótesis nula.

Entre estas, la característica de sexo y residencia (Urban-mujer), muestra una probabilidad de -0.0117 con un $\mathrm{p}$ significativo de $0.058^{*}$, edad de $71-80$ años muestra $-0.0071: 0.098^{*}$, educación (básica) alcanza 0.0147:0.052*; ocupación (particular) se representa por -0.0194:0.001*** ocupación (jubilado) muestra 0.0169:0.001***; principal sustento económico alcanza $-0.0153: 0.002^{* \star *} \mathrm{y}$, padecer enfermedad enlistada $0.0208: 0.006^{* * *}$.

Los resultados muestran que en promedio, ser mujer urbana disminuye la probabilidad de sentirse altamente insatisfecho con la vida en 0,0238 puntos porcentuales comparado con los hombres, este resultado resulta interesante aunque no es estadísticamente significativo a los valores estándares (10\%, $5 \%$ y $1 \%$ ). Sin embargo, al existir un término de interacción (vivir en el área urbana) que es significativo a un nivel del $10 \%$, el efecto de ser mujer en la probabilidad de estar altamente insatisfecho con la vida es diferente para cada valor de la variable urbano, confirmándose que existe una diferencia entre las mujeres residentes en el área urbana y aquellas que residen en el área rural. Para las mujeres, el residir en el área urbana podría asociarse con una menor probabilidad de estar altamente insatisfechas con la vida en comparación con las mujeres residentes en el sector rural. Por lo cual se puede afirmar que los hombres, en este caso, sí estarían satisfechos con su calidad de vida indiferentemente de residir en el área urbana o rural.

Respecto a los grupos etarios se obtiene que, en promedio, estar en el rango de 71-80 años disminuye la probabilidad de sentirse altamente insatisfecho $(-0.0155)$ con la vida en general que se traduce en 0,71 puntos porcentuales comparados con aquellos comprendidos entre los 60 y 70 años.

En cuanto a las variables del estado civil, ninguna de las categorías muestra un efecto estadísticamente significativo, en consecuencia, no se encontró relación alguna con la percepción que tienen los AM de su calidad de vida si es viudo, casado o posee alguna pareja. El mismo resultado se obtuvo para las variables que hacen referencia a la etnia declarada por los AM que no resultó significativa en ningún caso. Así mismo, respecto a las variables de educación se observa que en promedio, haber cursado la instrucción básica aumenta la probabilidad de sentirse altamente insatisfecho con la vida en general en 1,47 puntos porcentuales comparado con aquellos que han cursado instrucción de tercer nivel siendo esta diferencia estadísticamente significativa al $10 \%$. Esto quiere decir que aquellos que alcanzaron una educación superior perciben una mejor calidad de vida que aquellos que sólo aprobaron la educación primaria. 
Tabla 5: Efectos Marginales Promedio para la categoría Altamente insatisfecho

\begin{tabular}{|c|c|c|c|c|c|c|}
\hline Delta-method & $d y / d x$ & Error Estándar & $z$ & $p>|z|$ & \multicolumn{2}{|c|}{ (95\% Conf. Interval) } \\
\hline Mujer & -0.0002 & 0.0055 & -0.04 & 0.966 & -0.0111 & 0.0107 \\
\hline Urban-mujer & -0.0117 & 0.0061 & -1.89 & $0.058^{*}$ & -0.0238 & 0.0004 \\
\hline Edad $71-80$ años & -0.0071 & 0.0043 & -1.65 & $0.098^{*}$ & -0.0155 & 0.0013 \\
\hline Edad 81 + años & -0.0058 & 0.0049 & -1.18 & 0.239 & -0.0154 & 0.0038 \\
\hline Estado civil (soltero) & 0.0096 & 0.0090 & 1.07 & 0.286 & -0.0080 & 0.0273 \\
\hline Estado civil (divorciado) & 0.0091 & 0.0074 & 1.23 & 0.220 & -0.0054 & 0.0236 \\
\hline Estado civil (viudo) & 0.0031 & 0.0046 & 0.68 & 0.498 & -0.0060 & 0.0123 \\
\hline Etnia (afroamericano) & 0.0009 & 0.0113 & 0.09 & 0.932 & -0.2128 & 0.0232 \\
\hline Etnia (montubio) & 0.0067 & 0.0046 & 1.43 & 0.152 & -0.0024 & 0.015 \\
\hline Etnia (indígena) & -0.0099 & 0.0064 & -1.54 & 0.124 & -0.0225 & 0.0027 \\
\hline Etnia (blanco) & 0.0009 & 0.0382 & 0.03 & 0.980 & -0.0740 & 0.0760 \\
\hline Educación (ninguna) & 0.0176 & 0.0130 & 1.35 & 0.176 & -0.0078 & 0.0431 \\
\hline Educación (básica) & 0.0147 & 0.0076 & 1.94 & $0.052^{*}$ & -0.0001 & 0.0296 \\
\hline Educación (bachillerato) & 0.0085 & 0.0092 & 0.92 & 0.357 & -0.0096 & 0.0266 \\
\hline Ocupación (empresario) & 0.0192 & 0.0266 & 0.72 & 0.471 & -0.0329 & 0.07141 \\
\hline Ocupación (particular) & -0.0194 & 0.0059 & -3.29 & $0.001^{\star * *}$ & -0.0310 & -0.0078 \\
\hline Ocupación (publico) & -0.0058 & 0.0278 & -0.21 & 0.834 & -0.0603 & 0.0486 \\
\hline Ocupación (jubilado) & -0.0169 & 0.0053 & -3.19 & $0.001^{* * *}$ & -0.0273 & -0.0065 \\
\hline Ocupación (buscando empleo) & -0.0119 & 0.0100 & -1.20 & 0.232 & -0.0316 & 0.0076 \\
\hline Ocupación (en otra situación) & -0.0091 & 0.0058 & -1.55 & 0.120 & -0.0206 & 0.0023 \\
\hline Principal sustento económico & -0.0153 & 0.0050 & -3.03 & $0.002^{* \star *}$ & -0.0252 & -0.0054 \\
\hline Padecer enfermedad enlistada & 0.0208 & 0.0075 & 2.77 & $0.006^{\star \star *}$ & 0.0060 & 0.0355 \\
\hline Residente zona urbana & 0.0342 & 0.0351 & 0.97 & 0.330 & -0.0346 & 0.1030 \\
\hline Urbanos enfermedad & -0.0282 & 0.0259 & -1.09 & 0.277 & -0.0790 & 0.0226 \\
\hline
\end{tabular}

En general, las variables que recogen la ocupación del individuo resultan importantes y, a pesar que individualmente no son todas estadísticamente distintas de cero o significativas a los niveles estándares, en la prueba de significancia conjunta sí lo son, puesto que para dicho cálculo se realizó un test de significancia conjunta, y se obtuvo un $\mathrm{p}$ valor de 0,0051 ; es decir, significativa al $1 \%$. Por otra parte, en promedio, estar empleado por una institución privada o particular disminuye la probabilidad de sentirse altamente insatisfecho con la vida en general en 1,94 puntos porcentuales comparado con aquellos que laboran por cuenta propia. Así mismo, se encontró que, en promedio, el ser jubilado disminuye la probabilidad de sentirse altamente insatisfecho con la vida en general en 1,69 puntos porcentuales comparado con aquellos que laboran por cuenta propia. Esto implicaría que aquellos que aún laboran en relación de dependencia perciben una mejor calidad de vida que aquellos que laboran independientemente, esto podría ser a causa de la seguridad y estabilidad que perciben aquellos que reciben un sueldo mensual fijo a diferencia de aquellos que perciben ingresos variables, lo que explicaría también el hecho que aquellos adultos mayores jubilados se sientan más satisfechos.

Por otra parte, se observa que los jubilados tienen ciertas preferencias en acceso a servicios que podrían mejorar su calidad de vida, como son por ejemplo, la atención en hospitales públicos, a pesar de no aportar al Instituto Ecuatoriano de Seguridad Social (IESS) y, la devolución - exoneración de ciertos impuestos como el Impuesto al Valor Agregado (IVA).

Respecto a la situación de responsabilidad económica dentro del hogar, las estimaciones muestran que, en promedio, el ser la principal fuente de sustento económico disminuye la probabilidad de sentirse altamente insatisfecho con la vida en general en 1,53 puntos porcentuales comparado con aquellos que no son el principal sustento económico. Este resultado podría explicarse por el hecho que el tener responsabilidad económica dentro del hogar, a pesar de ser un adulto mayor, contribuiría en la autoestima del individuo pudiéndose traducir en valoraciones más altas en la auto-calificación de la calidad de vida percibida. En general, las personas que reportaron ninguna enfermedad alcanzó un 5,02\%, en consecuencia, el restante $94,98 \%$ sí reportó a lo menos una enfermedad de las listadas. 
Los resultados de la estimación muestran que, en promedio, el padecer de una o más de las enfermedades listadas en la encuesta, incrementa la probabilidad $\left(0.006^{* * *}\right)$ de sentirse altamente insatisfecho con la vida en general en 2,08 puntos porcentuales respecto de quienes no reportaron padecer dichas enfermedades. En consecuencia, como era de esperar, el padecer una enfermedad específica tendría una asociación negativa en la auto-calificación de la calidad de vida del individuo. Al respecto, existe evidencia que el padecimiento que perjudique la salud afecta de manera significativa el bienestar de los AM (Sempértegui et al., 2011; Sempértegui et al., 2006). El término de interacción (urbano) entre las variables enfermedad y área de residencia, no resulta estadísticamente significativo distinto de cero o a los niveles estándares de significancia $(0.330$ y 0.277$)$, resultado que sugiere que no existiría diferencia alguna entre los adultos mayores que reportan alguna enfermedad y el lugar urbano o rural de residencia.

\section{CONCLUSIONES}

1. La ocupación y nivel de educación de los AM muestra una asociación estadísticamente significativa en la auto-calificación de la calidad de vida. Los AM con menor nivel de educación son más propensos a sentirse menos satisfecho con su vida. Una posible alternativa para mejorar la percepción de satisfacción derivada de la educación, podría ser el incluirlos en programas de capacitación que, si bien es cierto, no culminarían con la obtención de un título oficial sí contribuirían a mejorar su auto percepción de calidad de vida.

2. La ocupación incide de manera inversa en la insatisfacción con la calidad de vida de los AM. En consecuencias, aumentaría la satisfacción siempre y cuando los AM se encontraren en una situación laboral de trabajar de manera particular o encontrarse jubilados. Esto generaría un tipo de incentivo emocional y económico que les permitiría tener acceso a un mayor nivel consumo, a una cierta estabilidad económica y laboral y, en el caso de los jubilados, a los beneficios sociales determinados por el Gobierno tales como acceso gratuito a los servicios de salud y ciertos programas de devoluciones y exoneraciones de impuestos.

3. En general el área de residencia no es una variable significativa para determinar la calidad de vida de los adultos mayores, sin embargo, el área de residencia sí juega un papel importante cuando se habla de las diferencias entre hombres y mujeres. Así los resultados indican que para el caso de las mujeres el residir en el área urbana se asociaría con una menor probabilidad de sentirse altamente insatisfecho con la vida, manteniendo el resto de variables fijas.

4. Finalmente, existen variables determinantes en la calidad de vida de los AM, tales como: padecer alguna enfermedad, ser el ingreso principal del hogar, ser jubilado, trabajar de manera particular, haber logrado un nivel de educación básica y pertenecer al rango de edad entre 71 y 80 años.

\section{REFERENCIAS}

Alonso, P., F. Sansó, A. Díaz-Canel, M. Carrasco, y T. Oliva, Envejecimiento poblacional y fragilidad en el adulto mayor, Rev. Cubana Salud Pública, Ciudad de La Habana, 33 (1) 1-17 (2007)

Asamblea Nacional del Ecuador. Constitución de la República del Ecuador, Montecristi. Registro Oficial 449 de 20-oct. 2008, (en línea: https://goo.gl/PzkmEQ. Acceso: 26 de enero de 2017), (2008)

Boz I. y C. Akbay, Factors influencing the adoption of maize in Kahramanmaras province of Turkey, Agricultural Economics, 33 (3) 431-440 (2005)

CEPAL. Comisión para América Latina y el Caribe, Older persons in Latin America and the Caribbean (en línea: https://goo.gl/3qq9BX. Acceso: 26 de enero de 2017), (2014)

CEPAL. Comisión para América Latina y el Caribe, Economic Commission for Latin America and the Caribbean. Long term population estimates and projections 1950-2100 (en línea: https://goo.gl/iMNgpH. Acceso: 26 de enero de 2017), (2015)

Choo S., G. Collantes y P. Mokhtarian, Wanting to travel, more or less: Exploring the determinants of the deficit and surfeit of personal travel, Transportation, 32 (2) 135-164 (2005)

CNII. Consejo Nacional para la Igualdad Intergeneracional ¿Quiénes y cuántos son? (en línea: https://goo.gl/Fqn8La. Acceso: 26 de enero de 2017), (2014)

Deaton, A., Income, health, and well-being around the world: Evidence from the Gallup World Poll, The journal of economic perspectives, 22(2) 53-72 (2008) 


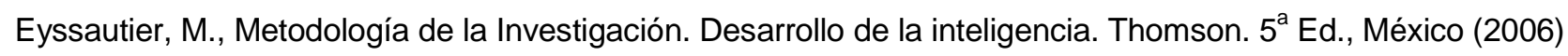

Fosgerau, M. y T.B. Bjorner, Joint models for noise annoyance and willingness to pay for road noise reduction, Transportation research Part B Methodological, 40(2) 164-178 (2006)

Freire, W., E. Rojas, L. Pazmiño, M. Fornasini, S. Tito. P. Buendía, Encuesta Nacional de Salud, Bienestar y Envejecimiento SABE I Ecuador 2009-2010 (en linea: https://goo.gl/APFcXb. Acceso: 26 de enero de 2017) MIES, INEC, (2010)

Greene, W., Econometric analysis. New York, Prentice Hall (2003)

Guarín A., A. Ramírez, F. Torres., Modelos Multinomiales: Un Análisis de sus Propiedades, Revista ingenierías Universidad de Medellín, ISSN 1692-3324, (en línea), 11 (20) 87-104, 2012 https://goo.gl/dLh97h. Acceso: 26 de enero de (2017)

Guedes A., y V. Martínez, Apuntes sobre: The problem of the social cost. eGesta, 6 (1) 46-59 (2010)

Hernández, R., C. Fernández, y P. Baptista, Metodología de la Investigación. México D. F.: Mc Graw-Hill, (2006)

Hritcu, S., A multilevel analyses of life satisfaction in Central and Eastern Europe. Procedia economics and finance, 20, 289-300 (2015)

Hyland, M.E., A.N. Jeffery y T.J. Wilkin, A biological, latent variable model of health (EarlyBird 68). doi: 10.1016/j.bbi.2014.02.018, Brain Behav. Immun., (en línea) 40, 104-109 (2014)

Knoepfel, P., C. Larrue, y F. Varone., Análisis y gestión de políticas públicas. Barcelona, Editorial Ariel (2012)

Malagón-Londoño G., R. Galán y G. Pontón, Administración hospitalaria. $3^{\text {a }}$ Ed., Bogotá: Editorial Médica Internacional (2008)

Mincu, C. y A. Tascu, Social support, satisfaction with physician-patient relationship, couple satisfaction, body satisfaction, optimism as predictors of life satisfaction in people having a current perceived health problem. Procedia - Social and Behavioral Sciences, 187, 772-776 (2015)

Ndjeunga J. y C.H. Nelson, Toward understanding household preference for consumption characteristics of millet varieties: a case study from western Niger, Agricultural Economics, 32(2) 151-165 (2005)

Osorio, P., M. J. Torrejón, y M. S. Anigstein, Calidad de vida en personas mayores en Chile, Revista Mad Universidad de Chile, 24, 61-75 (2011)

Penna, F., Modelo de Regresión Logística aplicada a niños con maloclusión dental. Fundamentos en Humanidades, I.S.S.N. 1668-7116, (en línea), Año VII; № I-II (13-14), 201-211, 2006, https://goo.gl/5RQbs8. Acceso: 26 de enero de (2017)

Penna, F., La utilización de variables indicadoras en un Modelo de Regresión Múltiple. Fundamentos en Humanidades, I.S.S.N. 1668-7116, (en línea), Año XI - Número II (22) 89-99, 2010, https://goo.gl/VP231w Acceso: 26 de enero de (2017)

Quintana, M. y T. Paravic, Calidad de vida en el trabajo del equipo de enfermería/Qualidade de vida no trabalho na equipe da enfermagem/Quality of work life in nursing staff. Revista Brasileira de Enfermagem, 67 (2), 302-305 (2014)

Rodríguez M. C., J.C., Cáceres. Modelos de elección discreta y especificaciones ordenadas: una reflexión metodológica, Estadística Española, 49(166) 451-471(2007)

Sempértegui F., B. Estrella, N. Elmieh, M. Jordan, T. Ahmed, A. Rodrıguez, K. L. Tucker, D. H. Hamer, P. G. Reeves, y S. N. Meydani, Nutritional, immunological and health status of the elderly population living in poor neighbourhoods of Quito, Ecuador. Br J. Nutr., 96, 845-53 (2006)

Sempértegui F., B. Estrella, K.L. Tucker, D.H. Hamer, X. Narvaez, M. Sempertegui, J.K. Griffiths, S.E. Noel, G.E. Dallal, J. Selhub, Metabolic syndrome in the elderly living in marginal peri-urban communities in Quito, Ecuador. doi: 10.1017/S1368980010002636. Public Health Nutr., (en línea), 14(5) 758-767 (2011) 
Solís C., R. Fantin, M. Kelly-Irving y C. Delpierre, Physiological wear-and-tear and later subjective health in mid-life: Findings from the 1958 British birth cohort. doi: 10.1016/j.psyneuen.2016.08.018. Psychoneuroendocrinology, (en línea) 74, 24-33 (2016)

United Nations, Department of Economic and Social Affairs, Population Division. World Population 2015, New York, NY 10017, Printed in the United States of America, 15-15398-December 2015 (en línea: https://goo.gl/MPDiVB. Acceso: 26 de enero de 2017)

Urzúa, A., Calidad de vida relacionada con la salud: Elementos conceptuales. https://goo.gl/c1ukTt. ISSN 0034-9887, Revista Médica de Chile, 138, 358-365 (2010)

Urzúa, A. y A. Caqueo-Urízar, Calidad de vida: Una revisión teórica del concepto. Terapia psicológica, 30(1), 61-71 (2012)

Urzúa, A., M. Bravo, M. Ogalde y C. Vargas, Factores vinculados a la calidad de vida en la adultez mayor. doi.org/10.4067/S0034-98872011000800005. Revista médica de Chile, (en línea), 139(8), 1006-1014 (2011)

Walpole, R., R. Myers, S. Myers, y K. Ye, Probabilidad y estadística para ingenierías y ciencias. México D.F. Editorial Pearson Educación (2007) 
